\title{
We should not avoid diclofenac in the treatment of spondyloarthritis
}

\author{
Gerhard Zingler ${ }^{1}$, Thomas Herdegen ${ }^{2}$ \\ ${ }^{1}$ Medical Department, Former employee of MSD Sharp \& Dohme GmbH, Munich, Germany; ${ }^{2}$ Institute of Experimental and Clinical Pharmacology, \\ University Hospital Schleswig-Holstein, Kiel, Germany \\ Correspondence to: Thomas Herdegen. Institute of Experimental and Clinical Pharmacology, University Hospital Schleswig-Holstein, Campus Kiel, \\ Hospitalstrasse 4, 24105 Kiel, Germany. Email: t.herdegen@pharmakologie.uni-kiel.de. \\ Comment on: Dubreuil M, Louie-Gao Q, Peloquin CE, et al. Risk of myocardial infarction with use of selected non-steroidal anti-inflammatory drugs \\ in patients with spondyloarthritis and osteoarthritis. Ann Rheum Dis 2018;77:1137-42.
}

Submitted Aug 27, 2018. Accepted for publication Sep 17, 2018.

doi: $10.21037 / \mathrm{atm} .2018 .09 .42$

View this article at: http://dx.doi.org/10.21037/atm.2018.09.42

Dubreuil et al. (1) report about the risk of myocardial infarction (MI) by NSAID in a nested-control study with patients suffering from spondyloarthritis (SpA). Current diclofenac use, but not naproxen use, was associated with an OR of 3.3 for MI when compared with remote use of any NSAID. Recent use of diclofenac had a lower risk (OR 1.40), whereas recent use of NSAID was not associated with increased MI outcome. In OA, the risk of diclofenac (OR 1.26) was not different with the risk of other NSAID (OR 1.17).

Within the SpA-cohort, MI cases had much more traditional MI risk factors as the controls, e.g., $38.3 \%$ suffering from ischaemic heart disease but only $8.6 \%$ in the controls, i.e., 4.5 -fold more. On the other hand, only $25 \%$ of SpA were on Aspirin-i.e., an obvious substantial under therapy (and most likely no sufficient treatment), but $15 \%$ of the controls (with only $8.6 \%$ ischemic heart disease). Most SpA patients (95\%) received more than $100 \mathrm{mg} / \mathrm{d}$ diclofenac, the maximum dosage reflecting a severe inflammatory status. Unfortunately, there was no further information on the SpA severity of diclofenac users compared with SpA patients on other NSAIDs. And most important, no data were given for the study populations of patients with diclofenac versus naproxen.

\section{The cardiovascular (CV) risk of NSAIDs in rheumatology and SpA}

Non-steroidal anti-inflammatory drugs (NSAIDs) are for a long time in use and count today to the worldwide most frequent used drugs. Because NSAIDs show a persuasive efficacy and are broadly available, these pain-mitigating drugs are essential elements in rheumatology. NSAIDs are indispensable in the treatment of acute and chronic pain as well as inflammation in cases of osteoarthritis (OA), rheumatoid arthritis (RA) and other arthritic conditions. OA and RA are the most common arthritic types in adults (2).

Although the gastrointestinal (GI) side effects of NSAIDs are known for a long time, the CV safety is discussed no more than circa 20 years ago. The point of origin for the discussion on CV safety of NSAIDs was the Vioxx ${ }^{\circledR}$ Gastrointestinal Outcomes Research (VIGOR) trial. This clinical trial was conceived to investigate the GI safety of rofecoxib and naproxen in patients with RA (3). The patients of the rofecoxib group had as expected significantly less GI events, and the death rates from CV events were almost identical in both groups, but the group of naproxen patients had remarkably less MIs (3).

This was the beginning of a discussion about the increased CV risk of NSAIDs which continues till this day. While primary only the COX-2 specific inhibitors (coxibs) were considered as fraught with $\mathrm{CV}$ risk, now the discussion about the CV risk on the whole class of NSAIDs has expanded. In the interim a variety of large clinical studies, reviews of the literature and meta-analyses of RCTs and observational studies, comparing one NSAID with another or with placebo confirmed the increased CV risk, while other examinations could identify no $\mathrm{CV}$ risk by NSAIDs in 
standard doses (4).

Meaningful epidemiological investigations have shown an increased risk of $\mathrm{CV}$ disease in patients with RA which have to take NSAIDs. The CV risk of these RA patients is affected by traditional CV risk factors as well as by disease activity. Besides, topical examinations confirm the important role of inflammation in the etiopathology of arteriosclerosis and the elevated CV risk of patients with RA (5).

An increased MI risk was always observed in recent years in several population-based studies of patients with multiple types of inflammatory arthritis. Besides, there was no remarkable difference in the incidence and prevalence of major cardiovascular events (MACE) between RA and AxSpa or PsA. Out of this, it can be concluded that inflammation determined the increased $\mathrm{CV}$ risk stronger than the specific type of the present arthritis $(6,7)$.

Due to their anti-inflammatory potency, NSAIDs remain the cornerstone treatment in $\mathrm{SpA}(8)$, and, although one supposes that these drugs increase the $\mathrm{CV}$ risk in the general population. However, there are only few data to the $\mathrm{CV}$ risk of NSAIDs in patients with inflammatory arthritis or autoimmune diseases (RA, SpA or PsA) (9).

In the linked study, Dubreuil and colleagues examined the risk of MI associated with use of NSAIDs in patients with $\mathrm{SpA}$, and also assessed risk among patients with OA, a non-inflammatory form of arthritis (1). The authors hypothesised that MI risk with specific NSAIDs would follow a similar pattern in patients with $\mathrm{SpA}$ as compared with that in the general population, but would be greater in SpA due to systemic inflammation.

\section{The evidence of the contrary: NSAIDs lower the CV risk in RA and SpA}

A competing theory is that NSAID use in inflammatory arthritis may protect against $\mathrm{CV}$ events by reducing systemic inflammation, which itself increases risk for MI.

This hypothesis was confirmed by several studies which have reported a lack of increase (10) or even a reduction of CVD rates in SpA patients treated with NSAIDs (11-13) and, in addition, a clear reduction of elevated acute phase reactants as well as also of serum proinflammatory cytokines $(14,15)$.

Bakland et al. (11) investigated standardized mortality ratios, causes of death and survival predictors in a large regional cohort of patients with AS. Factors independently associated with reduced survival were i.e., increasing levels of C-reactive protein (OR 2.68) and the non-use of any
NSAIDs (OR 4.35).

Tsai et al. (12) conclude that long-term frequent use of NSAIDs might protect AS patients from CVD; because the risks of MACE were significantly lower at 12 months-a trend showing that longer exposure correlated with lower risk. These results are also supported by examinations in patients with RA $(16,17)$.

\section{Does diclofenac truly increase the risk of $\mathrm{MI}$ in SpA?}

In contrast to these results, the authors of the current study found that use of current diclofenac in SpA was associated with twofold to threefold risk of MI relative to remote use of any NSAID. The relative risk associated with diclofenac in SpA differed from the risk in OA. Current naproxen use did not increase MI risk in SpA or OA (1).

It is very interesting that Dubreuil et al. (1) found important differences in relative risks across different NSAIDs in patient's populations with different diseases.

For example, Salpeter et al. (18) found in a metaanalysis no increased $\mathrm{CV}$ risk in patients with joint disease which have used a NSAID, but an indication for risk was present in trials of Alzheimer's disease (18) and in colonic adenoma (19). This was the first hint to the fact that the $\mathrm{CV}$ risk is not identical in different populations. Other investigations in patients with $\mathrm{OA}$ and patients with $\mathrm{RA}$ have confirmed this (20).

The current study report on differences, too. It is worth as secure that the absolute $\mathrm{CV}$ risk for the RA or $\mathrm{SpA}$ patients is higher but the relative $\mathrm{CV}$ risk associated with overall NSAID use was small and significantly lower in RA patients compared to controls (4,21-23). Dubreuil et al. (1) examined patients with $\mathrm{SpA}$ and with $\mathrm{OA}$ and found that use of current diclofenac conferred an increased relative risk for $\mathrm{CV}$ events compared to past use among both patients groups, but the relative risk was increased for $\mathrm{SpA}$ compared with OA.

The design (nested case-control study) of the present study is not likely to test the hypothesis underlying this study. The authors calculated a crude OR for the odds of current NSAID use relative to remote NSAID use for cases and controls. As already supposed, confounding by indication still remains a potential concern since NSAID prescription may indicate a period of pain or increased disease activity, and it may be that painful condition or disease activity that truly puts a subject at risk. A phase of high disease activity was compared therefore to a phase of 
low activity. But, a violent pain and an existing inflammation could be a confounding factor. Consequently, it can be assumed that patients with inflammation, aches and pains take more NSAIDs and for a longer time to relieve symptoms. It is well known that patients with inflammatory arthritis (RA, PsA, and SpA) have a measurably increased $\mathrm{CV}$ risk, and that this markedly elevated risk is the highest in those with high disease activity. Patients with an activated inflammatory arthritis present increased platelet function, specific to the ADP pathway. The boosted platelet overreaction to ADP can represent a pro-thrombotic bias in patients with inflammation and NSAID use $(4,24)$. Therefore, patients in the group with active inflammatory arthritis and platelet hyper-reactivity profit from the ability of naproxen to inhibit platelet aggregation in patients with activated RA $(4,25)$.

The authors of the present study calculated a crude OR for the odds of current NSAID use (within 180 days) relative to remote use ( $>365$ days) of any NSAID for cases and controls (1). Besides, the period of the current use of NSAID is too long and therefore, it cannot be concluded that MI are the result of NSAID use. In the majority of studies, the observation period prior to the index is no longer than 30 days (25). Moreover, CV events could be evoked by discontinuation of NSAID therapy.

RA patients differ in the current or past use of NSAIDs (25). For example, the ORs for current and past use of naproxen were 0.57 and 0.90 , respectively. Current use of diclofenac was associated with an elevated risk of MI but the ORs for past use of diclofenac was lower as for the current use. However, the risk was significantly increased with past use of naproxen in patients with RA, suggesting that any antithrombotic effect of naproxen occurs only under a current therapy and this is an effect on blocking platelet aggregation (25).

Dubreuil et al. (1) conclude: "Our results suggest that diclofenac use contribute to risk of MI in patients with SpA and clinicians that MIs could be prevented through preferential use of naproxen." The most confident data on these issues comes from large randomized controlled trials, such as the recently reported Prospective Randomized Evaluation of Celecoxib Integrated Safety Vs Ibuprofen Or Naproxen (PRECISION) study which tested different NSAIDs across patients with OA or RA (20).

The PRECISION trial looks at the CV safety of NSAIDs in more than 24,000 patients. The results showed a comparable $\mathrm{CV}$ risk profile for moderate doses of the selective COX-2 inhibitor celecoxib and the nonselective
NSAIDs ibuprofen and naproxen (20). In the OA group and the RA group; the risk of a major adverse CV event did not differ significantly between those randomized to receive celecoxib and those randomized to receive naproxen. An exploratory analysis also assessed a composite end point of all four major safety events, including major adverse $\mathrm{CV}$ events, composite serious GI events, serious renal events, and all-cause mortality. The HRs for the RA subgroup did not show any difference in the risk of major safety events between treatment arms. But, for patients with RA, risk for all-cause mortality was higher in the naproxen group than in the celecoxib group (20). A similar discrepancy, i.e., lower event rate of MI, however, a higher rate of $\mathrm{CV}$ death was observed for Naproxen too in other studies $(4,25)$.

The authors draw the conclusion that MI risk in SpA is increased among current users of diclofenac and should be preferentially treated with naproxen. Unfortunately, the authors did not provide any data on the safety of naproxen, in particular on its risk of GI bleeding and other events, which is dramatically enhanced compared to diclofenac (9). Moreover, there is no differential information of SpA-patients on diclofenac and on naproxen. Therefore, the conclusion to avoid diclofenac in SpA has to be critically discussed.

\section{Acknowledgements}

None.

\section{Footnote}

Conflicts of Interest: The authors have no conflicts of interest to declare.

\section{References}

1. Dubreuil M, Louie-Gao Q, Peloquin CE, et al. Risk of myocardial infarction with use of selected non-steroidal anti-inflammatory drugs in patients with spondyloarthritis and osteoarthritis. Ann Rheum Dis 2018;77:1137-42.

2. Sangha O. Epidemiology of rheumatic diseases. Rheumatology (Oxford) 2000;39 Suppl 2:3-12.

3. Bombardier C, Laine L, Reicin A, et al. Comparison of upper gastrointestinal toxicity of rofecoxib and naproxen in patients with rheumatoid arthritis. VIGOR Study Group. N Engl J Med 2000;343:1520-8.

4. Zingler G, Hermann B, Fischer T, et al. Cardiovascular adverse events by non-steroidal anti-inflammatory drugs: 
when the benefits outweigh the risks. Expert Rev Clin Pharmacol 2016;9:1479-92.

5. Lauper K, Gabay C. Cardiovascular risk in patients with rheumatoid arthritis. Semin Immunopathol 2017;39:447-59.

6. Schieir O, Tosevski C, Glazier RH, et al. Incident myocardial infarction associated with major types of arthritis in the general population: a systematic review and meta-analysis. Ann Rheum Dis 2017;76:1396-404.

7. Lauper K, Courvoisier DS, Chevallier P, et al. Incidence and prevalence of major adverse cardiovascular events in rheumatoid arthritis, psoriatic arthritis, and axial spondyloarthritis. Arthritis Care Res (Hoboken) 2018. [Epub ahead of print].

8. van der Heijde D, Ramiro S, Landewé R, et al. 2016 update of the ASAS-EULAR management recommendations for axial spondyloarthritis. Ann Rheum Dis 2017;76:978-91.

9. Bhala N, Emberson J, Merhi A, et al. Vascular and upper gastrointestinal effects of non-steroidal anti-inflammatory drugs: meta-analyses of individual participant data from randomised trials. Lancet 2013;382:769-79.

10. Rivière E, Etcheto A, den Bosch FV, et al. THU0350 Effect of nsaid consumption on cardiovascular events in spondyloarthritis. Ann Rheum Dis 2017;76:336.

11. Bakland G, Gran JT, Nossent JC. Increased mortality in ankylosing spondylitis is related to disease activity. Ann Rheum Dis 2011;70:1921-5.

12. Tsai WC, Ou TT, Yen JH, et al. Long-term frequent use of non-steroidal anti-inflammatory drugs might protect patients with ankylosing spondylitis from cardiovascular diseases: a nationwide case-control study. PLoS One 2015;10:e0126347.

13. Haroon NN, Paterson JM, Li P, et al. Patients with ankylosing spondylitis have increased cardiovascular and cerebrovascular mortality: a population-based study. Ann Intern Med 2015;163:409-16.

14. Kroon F, Landewé R, Dougados M, et al. Continuous NSAID use reverts the effects of inflammation on radiographic progression in patients with ankylosing spondylitis. Ann Rheum Dis 2012;71:1623-9.

15. Yan Y, Guo TM, Zhu C. Effects of nonsteroidal antiinflammatory drugs on serum proinflammatory cytokines in the treatment of ankylosing spondylitis.Biochem Cell

Cite this article as: Zingler G, Herdegen T. We should not avoid diclofenac in the treatment of spondyloarthritis. Ann Transl Med 2018;6(Suppl 1):S36. doi: 10.21037/atm.2018.09.42
Biol 2018;96:450-6.

16. Goodson NJ, Brookhart AM, Symmons DP, et al. Nonsteroidal anti-inflammatory drug use does not appear to be associated with increased cardiovascular mortality in patients with inflammatory polyarthritis: results from a primary care based inception cohort of patients. Ann Rheum Dis 2009;68:367-72.

17. Franklin J, Farragher TM, Lunt M, et al. Excess risk of hospital admission for cardiovascular disease within the first 7 years from onset of inflammatory polyarthritis. Ann Rheum Dis 2010;69:1660-4.

18. Salpeter SR, Gregor P, Ormiston TM, et al. Meta-analysis: cardiovascular events associated with nonsteroidal antiinflammatory drugs. Am J Med 2006;119:552-9.

19. Scott PA, Kingsley GH, Smith CM, et al. Non-steroidal anti-inflammatory drugs and myocardial infarctions: comparative systematic review of evidence from observational studies and randomised controlled trials. Ann Rheum Dis 2007;66:1296-304.

20. Solomon DH, Husni ME, Wolski KE, et al. Differences in Safety of Nonsteroidal Antiinflammatory Drugs in Patients With Osteoarthritis and Patients With Rheumatoid Arthritis: A Randomized Clinical Trial. Arthritis Rheumatol 2018;70:537-46.

21. Sfikakis PP, Bournia VK, Kitas G. Do non-steroidal antiinflammatory drugs increase or decrease cardiovascular risk in patients with rheumatoid arthritis? Clin Exp Rheumatol 2014;32:S-8-9.

22. Herdegen T. Cardioprotective Effect of NSAIDs. Dtsch Arztebl Int 2017;114:559.

23. Lindhardsen J, Gislason GH, Jacobsen S, et al. Nonsteroidal anti-inflammatory drugs and risk of cardiovascular disease in patients with rheumatoid arthritis: a nationwide cohort study. Ann Rheum Dis 2014;73:1515-21.

24. Mac Mullan PA, Peace AJ, Madigan AM, et al. Platelet hyper-reactivity in active inflammatory arthritis is unique to the adenosine diphosphate pathway: a novel finding and potential therapeutic target. Rheumatology (Oxford) 2010;49:240-5.

25. Watson DJ, Rhodes T, Cai B, et al. Lower risk of thromboembolic cardiovascular events with naproxen among patients with rheumatoid arthritis. Arch Intern Med 2002;162:1105-10. 